Tatiana Drevytska' / Roman Morhachov' / Lesya Tumanovska' / Georgiy Portnichenko' / Vasyl Nagibin' / Oleksiy Boldyriev' / Tatiana Lapikova-Bryhinska' / Veronika Gurianova' / Borys Dons'koi ${ }^{2}$ / Maxim Freidin 3 / Vladimir Ivanisenko4 / Elena Yu Bragina ${ }^{3}$ / Ralf Hofestädt ${ }^{5,6}$ / Victor Dosenko ${ }^{7}$

\title{
shRNA-Induced Knockdown of a Bioinformatically Predicted Target IL10 Influences Functional Parameters in
} \section{Spontaneously Hypertensive Rats with Asthma}

${ }^{1}$ Department of Ceneral and Molecular Pathophysiology, Bogomoletz Institute of Physiology, National Academy of Sciences of Ukraine, Kyiv 01024, Ukraine

${ }^{2}$ National Academy of Medical Sciences, State Institution “Institute of Pediatrics, Obstetrics and Gynecology", Kyiv, Ukraine

${ }^{3}$ Research Institute of Medical Cenetics, Tomsk NRMC, Tomsk, Russia. https://orcid.org/0000-0002-1103-3073.

4 Institute of Cytology and Genetics, Siberian Branch, Russian Academy of Sciences, Novosibirsk, Russia

${ }^{5}$ Bielefeld University, International Research Training Croup "Computational Methods for the Analysis of the Diversity and Dynamics of Cenomes", Bielefeld, Cermany

${ }^{6}$ Bielefeld University, Technical Faculty, AG Bioinformatics and Medical Informatics, Bielefeld, Germany

7 Department of Ceneral and Molecular Pathophysiology, Bogomoletz Institute of Physiology, National Academy of Sciences of Ukraine, 4 Bogomoletz str, Kyiv 01024, Ukraine, E-mail: dosenko@biph.kiev.ua

\begin{abstract}
:
One of the most common comorbid pathology is asthma and arterial hypertension. For experimental modeling of comorbidity we have used spontaneously hypertensive rats with ovalbumin (OVA)-induced asthma. Rats were randomly divided into three groups: control group, OVA-induced asthma group; OVA-induced asthma + IL10 shRNA interference group. Target gene (IL10) was predicted by ANDSystem. We have demonstrated that RNA-interference of IL10 affected cardiovascular (tested using Millar microcatheter system) as well as respiratory functions (tested using force-oscillation technique, Flexivent) in rats. We have shown that during RNA-interference of IL10 gene in vivo there were changes in both cardiac and lung function parameters. These changes in the cardiovascular parameters can be described as positive. But the more intensive heart workload can lead to exhaust and decompensation of the heart functions. Knockdown of IL10 gene in asthma modeling induces some positive changes in respiratory functions of asthmatic animals such as decreased elastance and increased compliance of the lungs, as well as less pronounced pathomorphological changes in the lung tissue. Thus, we provide the data about experimentally confirmed functionality changes of the target which was in silico predicted to be associated with both asthma and hypertension - in our new experimental model of comorbid pathology.
\end{abstract}

Keywords: asthma, arterial hypertension, comorbid pathology, IL10

DOI: $10.1515 /$ jib-2018-0053

Received: July 27, 2018; Revised: November 13, 2018; Accepted: November 13, 2018

\section{Introduction}

Since the works of Feinstein [1], Kraemer and van den Akker [2], [3] comorbidity or multi-morbidity is still in focus of many investigators worldwide [4]. One of the most often combinations of chronic diseases is asthma and arterial hypertension [5] or other cardiovascular pathology [6], [7]. It is not surprising because modern understanding of inflammation is beyond the classical concept of this pathological process as a response to infection and injuries, and the significance of low-grade chronic inflammation has now been established in the pathogenesis of non-inflammatory diseases (e.g. atherosclerosis, metabolic syndrome, arterial hypertension, obesity) has now been established [8], [9], [10], [11], [12], [13]. 
The main problem of comorbidity in clinics is poor prognosis. Polypharmacy is the next significant point: patients with two or more pathologies should receive simultaneously a lot of drugs leading to combination of side effects. Thus, it is important to find molecular targets that can be used for prospective treatment of both diseases with a single drug. Bioinformatical approaches in this situation are optimal considering huge volume of heterogeneous data for analysis. In our previous article, we used ANDSystem for the reconstruction and analysis of gene networks associated with both asthma and hypertension [14]. The gene network for asthma included 755 genes/proteins and 62603 interactions, while the gene network for hypertension - 713 genes / proteins and 45479 interactions. According to these date the genes encoding Interleukin-10 (IL10), Toll-like receptor 4 (TLR4), and catalase (CAT) have the highest priority in the development of asthma and arterial hypertension comorbidity.

In current research we demonstrate the influence of IL10 knockdown on development of experimental comorbidity of asthma and hypertension. RNA-interference of IL10, a gene predicted by ANDSystem, affected cardiovascular as well as respiratory functions are changed in spontaneously hypertensive rats with ovalbumininduced asthma.

\section{Methods}

\subsection{Animals}

All procedures followed the criteria, technical standards and rights applied to animal research. All trials were followed in accordance with the statements of the European Union regarding the handling of experimental animals. This investigation conformed to the laws and local ethical committee guidelines for animal research.

Spontaneously hypertensive male rats (SHR) aged 4-5 months (body mass 300-350 g) used in our study, were purchased from "Charles River Laboratories" company. Prior to experiments, rats were maintained in an animal facility under standard laboratory conditions for 3 months with free access to food and water. Hypertensive status was confirmed by measuring the blood pressure using non-invasive Mouse-Rat Blood Pressure Monitor - CODA (Kent Scientific, USA) before the start of the experiments. All measurements were provided after 10 min holding non stressed animal in special cage. Only animals with a systolic pressure $190.1 \pm 17.62$ $\mathrm{mmHg}$ and diastolic pressure $133.7 \pm 22.7 \mathrm{mmHg}$ were taken to experiment. Rats were randomly divided into three groups: control group, ovalbumin (OVA)-induced asthma group + scrambled shRNA-producing plasmid group; OVA-induced asthma + anti-IL10 shRNA-producing plasmid shRNA interference group. Each group included seven rats.

\subsection{Ovalbumin-Induced Asthma Model}

We established asthma model according to the methods of Li and Vanacker [15], [16] with some modifications. Rats were sensitized by intraperitoneal injection of $1 \mathrm{mg}$ OVA (S7951, Sigma-Aldrich, USA) adsorbed to $0.3 \mathrm{~mL}$ of $\mathrm{Al}(\mathrm{OH})_{3}$ as an adjuvant (Aluminum hydroxide Gel, colloidal suspension, Serva, Germany) in a total volume of $0.8 \mathrm{~mL}$ sterile normal saline solution on days 1 and 8 . Then starting from the day 15 , rats were challenged by intranasal exposure to $100 \mu \mathrm{L}$ of $1 \%$ OVA (in normal saline) once a day for 7 days. The control group was treated only with normal saline injections and intranasal delivery of saline solution. Euthanasia $(1.5 \mathrm{~g} / \mathrm{kg}$ urethane, intraperitoneally) was performed 1 day after the last intranasal delivery.

\subsection{Construction of shRNA-Expressing Vector}

shRNA sequence complementary to site on IL10 mRNA was designed in silico by siDESIGN Center (Dharmacon, a Horizon Discovery Group Co). We used BLAST (NCBI NIH, USA) algorithm to check it for unspecific binding. For 21 nucleotide RNA sequence, we generated 55 nucleotide sense and antisense oligonucleotides designed according to the recommendation of pSilencer 4.1-CMV neo vector kit (Ambion, ThermoFisher Scientific, USA) of with restriction sites for HindIII and BamHI to obtain sticky ends. These oligonucleotides were synthesized by Metabion, Germany. Sequences of both oligos are presented in Table 1. Two oligos were annealed and then immediately ligated into pSilencer 4.1-CMV neo vector (Ambion, ThermoFisher Scientific, USA). Ligation mix was transformed into chemically competent XL E. coli cells. Single colonies from solid LB agar medium were cultivated in LB broth overnight. Up to $5 \mathrm{mg}$ of plasmid DNA was purified with GeneJet Miniprep Kit (ThermoFisher Scientific, USA) from each colony to screen for successful ligation transformants. 
Ligation was confirmed by restriction analysis with HindIII and EcoRI and further electrophoresis analysis. Confirmed colonies were cultivated to make preparation-size purifying of shRNA vector. We used GeneJet Maxiprep Kit to obtain up to $1 \mathrm{mg}$ of pDNA which was dissolved in sterile endotoxin-free deionized water (ThermoFisher Scientific, USA) in sterile conditions and was ready for further experiments. The same procedure was performed for scrambled shRNA-encoding plasmid.

Table 1: Sequences of sense and antisense oligonucleotides.

\begin{tabular}{ll}
\hline Gene & Sequences \\
\hline IL10, NM_012854.2 & Top: 5'-GATCCGCTGAAGACCCTCTGGATACATTCAAGAGATGTATC \\
& CAGAGGGTCTTCAGCA-3' \\
& Bottom: 5'-AGCTTGCTGAAGACCCTCTGGATACATCTCTTGAATGTA \\
& TCCAGAGGGTCTTCAGCG-3' \\
Scrambled & Top: 5'-GATCCTTCTCCGAACGTGTCACGTTTCAAGAGAACGTGACA CGTTCGGAGAAA-3' \\
& Bottom: 5'-AGCTTTTCTCCGAACGTGTCACGTTCTCTTGAAACGTGA \\
& CACGTTCGGAGAAG-3' \\
\hline
\end{tabular}

\subsection{RNA Interference}

For IL10 knockdown in vivo, $100 \mu \mathrm{g}$ of the anti-IL10 shRNA-expressing plasmid was injected to rats via the tail vein on the $15^{\text {th }}$ day after the first injection of OVA. The same dose of scrambled plasmid was injected to the rats of the control group.

The effectiveness of RNA interference was measured using real-time PCR in the heart tissue.

\subsection{RNA Isolation, Reverse Transcription and Real-Time PCR}

Total RNA was isolated with Trizol reagent (Invitrogen, USA) reagent. RNA concentration was determined using the NanoDrop spectrophotometer ND1000 (NanoDrop Technologies Inc, USA). cDNA was synthesized from $5 \mu \mathrm{g}$ of total RNA by reverse transcription with $10 \mathrm{mM}$ Tris- $\mathrm{HCl}$ (pH 9.0), $5 \mathrm{mM} \mathrm{MgCl} 2 ; 1 \mathrm{~mm} \mathrm{dNTPs} ; 20 \mathrm{U}$ Ribo-Lock, Random hexamer primers $(0.5 \mu \mathrm{g} / \mu \mathrm{L})$ and $200 \mathrm{U}$ RevertAid H Minus M-MuLV Reverse Transcriptase. PCR was performed using the Applied Biosystems 2700, PerkinElmer, USA. Gene expression of IL-10 (Custom TaqMan ${ }^{\circledR}$ Gene Expression Assays, Rn01483988_g1) was determined using TaqMan ${ }^{\circledR}$ Gene Expression Assay ("Applied Biosystems", USA). The pairs of forward and reverse primers for above mentioned genes and the TaqMan probes for the target mRNAs were designed based on the rat mRNA sequence by Applied Biosystems (USA). Gene expression in each probe was normalized by GAPDH, using TaqMan Rodent GADPH Control Reagent (VIC ${ }^{\mathrm{TM}}$ Probe, lot number 0608014). The thermal cycles of PCR amplification were the following: initial denaturation step at $95{ }^{\circ} \mathrm{C}$ for $20 \mathrm{~s}$, followed by 60 cycles of treatment at $95{ }^{\circ} \mathrm{C}$ for $3 \mathrm{~s}$, and at $60^{\circ} \mathrm{C}$ for $30 \mathrm{~s}$ using 7500 Fast Real-time PCR (Applied Biosystems, USA). Analysis of obtained data was carried out with 7500 Fast Real-time PCR Software.

\subsection{Haemodynamic Analyses}

The evaluation of hemodynamic parameters was performed as described previously [17]. Briefly: rats were anesthetized with urethane $(1.5 \mathrm{~g} / \mathrm{kg}$, intraperitoneally), fixed, and the right carotid artery was dissected. An ultra-small catheter 2F (Millar Instruments, USA) was introduced retrogradely through the right carotid artery and the signals of pressure and volume in the left ventricle during the cardiac cycle were simultaneously recorded with visualization of $\mathrm{P}-\mathrm{V}$ curves. Recordings of the cardiodynamic parameters were performed in two modes: in an initial state and during temporary occlusion of the abdominal vena cava (for 7-10 s). We recorded the following cardio-hemodynamic parameters: heart rate (HR), end-systolic pressure (ESP), enddiastolic pressure, end-systolic volume of the left ventricle (ESV), end-diastolic volume of the left ventricle (EDV), stroke volume, cardiac output, ejection fraction, $\mathrm{dP} / \mathrm{dt} \max , \mathrm{dP} / \mathrm{dt} \min , \mathrm{dV} / \mathrm{dt} \max , \mathrm{dV} / \mathrm{dt}$ min and PdVdt max. Effective arterial elastance, determined according to the data of Sunagawa, was calculated as the ratio of ESP derived from the pressure-volume curve in the initial state to stroke volume. The pressure and volume ratio in the left ventricle was analyzed using the PVAN 3.6 program (Millar Instruments, Gulf Fwy, Houston, USA) with the conversion of relative volume units (RVU) to absolute units of volume ( $\mu \mathrm{L})$ using the 
data acquired during calibration procedure performed according to manufacturer recommendations formula (slope $20.25 \times$ RVU-intercept 29.05).

\subsection{Assessment of Airway Function}

Measurements of respiratory system resistance and compliance were measured using a SciReq FlexiVent ventilator (Scireq, Montreal, PQ, Canada). Briefly, $24 \mathrm{~h}$ after final challenge, animals were anaesthetized (urethane, $1.5 \mathrm{~g} / \mathrm{kg}$ ), tracheotomized (19Fr blunt Luer cannula), and connected to the ventilator. Animals were maintained at $37^{\circ} \mathrm{C}$ and, under muscle paralysis (Atracurium-Novo, NovoPharm Biosynthes, Ukraine). Aerosol challenge was performed after four successive baseline measurements using solutions of 4, 8, 16,32, or $64 \mathrm{mg} / \mathrm{kg}$ metacholine (MCh, A2251, Sigma-Aldrich, USA) or saline (as a control). All the mentioned solutions were delivered for $60 \mathrm{~s}$ each by an ultrasonic nebulizer (Aeroneb Pro, ANP-1100, Aerogen Ltd, Ireland) via the inspiratory port of the respirator. Before each delivery, two inspiratory phases were superimposed to standardize the volume history. Measurements were started $20 \mathrm{~s}$ after completion of each aerosol challenge, and eight successive recordings were collected during every $20 \mathrm{~s}$. The next dose was administered $60 \mathrm{~s}$ after the last recording. Resistance $\left(\mathrm{cmH}_{2} \mathrm{O}^{*} \mathrm{~s} / \mathrm{mL}\right)$ and compliance of respiratory system $\left(\mathrm{Crs}, \mathrm{mL} / \mathrm{cmH}_{2} \mathrm{O}\right)$ were calculated using FlexiWare 7.4 software (Scireq, Montreal, PQ, Canada).

\subsection{Measurement of Cytokine IL4 in CD4 ${ }^{+}$Cells in Bronchoalveolar Lavage Fluid (BALF)}

After insertion of a catheter into the trachea, the lungs of the animal were lavaged with $1.5 \mathrm{~mL}$ of Hanks balanced salt solution (Invitrogen, USA) [18]. The lavage fluid was centrifuged at $250 \mathrm{~g}$ during $3 \mathrm{~min}$, and the cell pellet was resuspended in $1.5 \mathrm{~mL}$ RPMI (Invitrogen) and the total cells were counted under a microscope (Olympus, Japan). The cells were then fluorescence-labeled for flow cytometric analysis in order to detect the percentage of T2 helpers in BALF. First of all, samples were incubated in RPMI 1640 for $4 \mathrm{~h}$ with ionomycin $(1 \mu \mathrm{g} / \mathrm{mL})$, brefeldin-A $(10 \mu \mathrm{g} / \mathrm{mL})$, and $25 \mathrm{ng} / \mathrm{mL}$ phorbol 12-myristate 13-acetate (all from Sigma, St Louis, MO, USA). Cells were washed by PBS and pellets were resuspended in $100 \mu \mathrm{L}$ PBS and stained with fluorescein isothiocyanate (FITC)-conjugated anti-CD4 (554837, Becton Dickinson, San Diego, CA, USA) for 30 min. The cells were lysed (Lysing Solution; BD Biosciences), permeabilized (Permeabilizing Solution; BD Biosciences), and stained with fluorochrome-conjugated mAbs. Cell pellets were resuspended in PBS and stained with fluorescein isothiocyanate (FITC)-conjugated anti-CD4 (554837, Becton Dickinson, San Diego, CA, USA) and Rphycoerythrin-conjugated anti-IL4 (555082, Becton Dickinson, San Diego, CA, USA) antibodies. The samples were stained for $30 \mathrm{~min}$ at room temperature in the dark. Then the cell suspensions were centrifuged, and the cell pellets were resuspended in PBS containing sodium azide and paraformaldehyde. Then, the cells were analyzed with the FACSCalibur flow cytometer (BD Biosciences, USA) equipped with CellQuest software (BD Biosciences, USA) [19].

\subsection{Morphological Analysis}

For light microscopic study, lung specimens were fixed in $10 \%$ buffered formalin and 5-mm thick paraffin sections were prepared and stained with hematoxylin and eosin (H\&E). H\&E-stained tissue sections were examined light-microscopically with Nikon Eclipse E200 microscope and Nikon DS-SI 1 camera and analyzed by using the ImageJ software. Morphometric measurements were made in lung tissue samples of five rats from each experimental group (in 10 nonoverlapping microscopic fields for each rat). The area of infiltrates content was measured at a magnification of $\times 100$ for each specimen and was expressed as a percentage relative to the area of the standard measuring frame.

The structural changes in the bronchiole walls of different size were analyzed as described previously [20]. The total bronchial wall area $(\mathrm{WAt}=\mathrm{Ao}-\mathrm{Abm})$, the inner wall area $(\mathrm{WAi}=\mathrm{Amo}-\mathrm{Abm})$ and the outer wall area $(\mathrm{WAo}=\mathrm{Ao}-\mathrm{Amo})$ were calculated based on the measured values of the basement membrane length of the epithelium ( $\mathrm{Pbm}$ ), the perimeter of the outer boundary of the smooth muscle (Pmo), the outer adventitial perimeter $(\mathrm{Po})$ and the areas defined by these parameters (Abm - basement membrane area, Amo - outer muscle area and Ao - outer airway area). The studied airways in each experimental group of rats were divided into two categories according to the length of their basement membrane: $\mathrm{Pbm}<1 \mathrm{~mm}$ (small), $1 \mathrm{~mm} \leq \mathrm{Pbm}<2 \mathrm{~mm}$ (medium). For analysis of the morphometric measurements the values of WAt, WAi, WAo, were normalized to $\mathrm{Pbm}$. 


\subsection{Statistical Analysis}

All data were checked for normality of distribution using Shapiro-Wilk normality test. Difference between groups was estimated using the Kruskal-Wallis rank sum test. Results were considered statistically significant if $p<0.05$. In cases when more then two groups were compared Kruskal-Wallis was followed by Dunn's test of multiple comparisons and the null hypothesis was rejected if $p \leq 0.025$.

\section{Results}

\subsection{Changes in mRNA Expression Upon Silencing of IL10}

Intravenously injection of IL10-specific shRNA-expressing plasmid induced a 28.6-fold downregulation of the IL10 mRNA level in the thymus and in 5.3-fold in spleen tissues $(p<0.05)$ compared with scrambled shRNAexpressing plasmid (Figure 1).

A

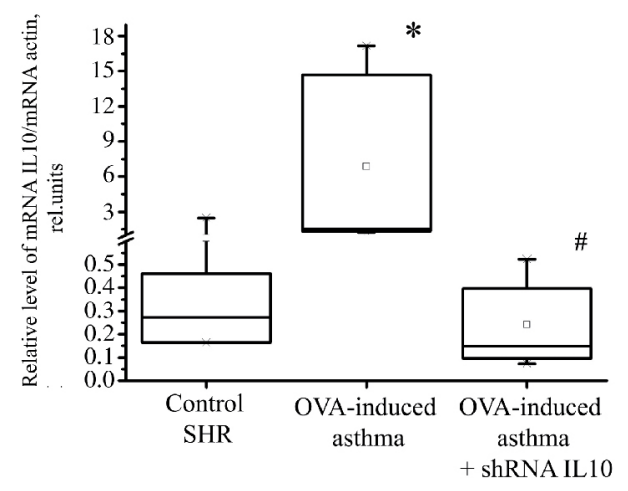

B

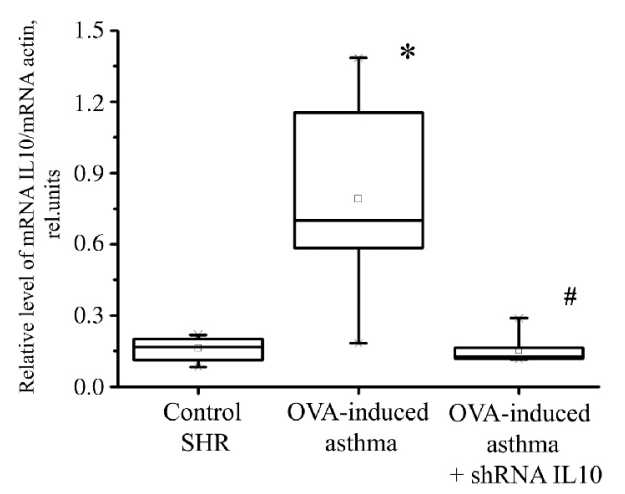

Figure 1: Relative level of IL10 mRNA expression in thymus tissue (A) and spleen tissue (B) in control SHR, in SHR with OVA-induced asthma + scrambled shRNA and in SHR with OVA-induced asthma + IL10 shRNA injection $(n=7$ per group). * Significant difference compared to control SHR, $p<0.05$. \# Significant difference compared to SHR with OVAinduced asthma + scrambled shRNA group, $p<0.05$.

\subsection{Changes in Cardio-Hemodynamic Parameters}

Basic parameters of heart function that were estimated using microcatheterisation of the left ventricle are presented in Table 2. In rats with OVA-induced asthma the next indicators were significantly lower than in control: heart rate, maximum volume, minimum volume, end-systolic volume, end-diastolic volume, Tau_w, but at the same time the main parameters of pump function were higher - stroke volume (on 27\%, $p<0.05$ ) and ejection fraction (on $63 \%, p<0.05$ ). Rats treated with anti-IL10-shRNA-expressing plasmid demonstrated difference of the same parameters with more pronounced elevation of stroke volume (on $45 \%, p<0.05$ ) and ejection fraction (on 76\%, $p<0.05$ ). Moreover, cardiac output (on 17\%, $p<0.05$ ), stroke work (on 1.9-fold, $p<0.05$ ), maximal power (on 1.83-fold, $p<0.05$ ) and main indicator of contractility Emax (on 3.1-fold, $p<0.05$ ) were significantly higher in this group compared to control rats. Knockdown of IL10 gene in asthma modeling in SHR induced the decrease of arterial elastance on $34 \%(p<0.05)$ that showed the decrease of the arterial vessels resistance. Time of diastolic relaxation (Tau_w) of the heart was also decreased in these animals (on $34 \%$ compared to control, $p<0.05)$.

Table 2: Cardiovascular parameters of control rats, OVA-induced asthma rats and OVA-induced asthma rats with IL-10 RNA interference.

\begin{tabular}{lrrr}
\hline Parameter & Control & $\begin{array}{r}\text { OVA-induced } \\
\text { asthma }\end{array}$ & $\begin{array}{r}\text { OVA-induced } \\
\text { asthma + IL-10 RNA } \\
\text { interference }\end{array}$ \\
\hline Heart rate (beats $/ \mathrm{min})$ & $397.4 \pm 1.51$ & $294.5 \pm 48.96^{\mathrm{a}}$ & $315.6 \pm 21.89^{\mathrm{a}}$ \\
Maximum volume $(\mu \mathrm{L})$ & $586.4 \pm 24.03$ & $450.7 \pm 24.03^{\mathrm{a}}$ & $482.7 \pm 81.79^{\mathrm{a}}$ \\
Minimum volume $(\mu \mathrm{L})$ & $399.8 \pm 23.01$ & $248.1 \pm 100.0^{\mathrm{a}}$ & $211.2 \pm 66.15^{\mathrm{a}}$
\end{tabular}


End-systolic volume ( $\mu \mathrm{L})$

End-diastolic volume $(\mu \mathrm{L})$

End-systolic pressure $(\mathrm{mmHg})$

Stroke volume $(\mu \mathrm{L})$

Ejection fraction (\%)

Cardiac output (mL/min)

Stroke work $(\mathrm{mmHg} / \mu \mathrm{L})$

Arterial elastance $(\mathrm{mmHg} / \mu \mathrm{L})$

dPdt max (mmHg/s)

dPdt min (mmHg/s)

Tau_w (ms)

Tau_g (ms)

Maximal power $(\mathrm{mW})$

Emax
$415.9 \pm 18.74$

$565.7 \pm 25.55$

$110.5 \pm 4.17$

$186.6 \pm 7.01$

$31.9 \pm 1.49$

$74.2 \pm 2.79$

$13430.11 \pm 1337.55$

$$
\begin{array}{r}
0.59 \pm 0.032 \\
5790.3 \pm 221.40 \\
-6640.8 \pm 146.81 \\
15.8 \pm 0.92 \\
13.8 \pm 0.35 \\
89.6 \pm 13.76 \\
2.79 \pm 0.29
\end{array}
$$

$$
\begin{array}{r}
271.9 \pm 105.36^{\mathrm{a}} \\
423.7 \pm 82.09^{\mathrm{a}} \\
109.6 \pm 20.99 \\
236.3 \pm 51.61^{\mathrm{a}} \\
52.3 \pm 16.0^{\mathrm{a}} \\
70.7 \pm 17.31
\end{array}
$$

$17635.03 \pm 8629.781$

$0.50 \pm 0.13$

$8287.1 \pm 4636.45$

$-5640.5 \pm 1786.94$

$10.4 \pm 1.91^{\mathrm{a}}$

$16.3 \pm 5.74$

$112.2 \pm 61.35$

$8.02 \pm 7.28$
$237.3 \pm 74.33^{\mathrm{a}}$

$453.9 \pm 110.06^{\mathrm{a}}$

$102.8 \pm 21.23$

$271.5 \pm 78.02^{\mathrm{a}}$

$56.2 \pm 6.61^{\mathrm{a}}$

$86.86 \pm 30.199^{a}$

$25994.59 \pm$

$12651.43^{\mathrm{a}, \mathrm{b}}$

$0.39 \pm 0.074^{\mathrm{a}, \mathrm{b}}$

$14102.7 \pm 5770.27$

$-6170.3 \pm 791.73$

$9.97 \pm 1.59^{\mathrm{a}}$

$18.8 \pm 6.87$

$164.3 \pm 73.81^{\mathrm{a}}$

$8.77 \pm 5.45^{\mathrm{a}}$

${ }^{\mathrm{a}}$ Means are statistically different against control group. ${ }^{\mathrm{b}}$ Means are statistically different against asthma group.

\subsection{Changes of Airway Function}

In control rats baseline resistance was $0.21 \pm 0.036$ and slightly increased substantially with methacholine from $0.43 \pm 0.041$ to $1.67 \pm 0.077 \mathrm{cmH}_{2} \mathrm{O}^{*} \mathrm{~s} / \mathrm{mL}$ (Figure 2A). In OVA-induced asthma group baseline resistance was 3.8 times higher $(p<0.05)$ comparing to this parameter in control animals, and increased to a greater extent with methacholine from $0.81 \pm 0.092$ to $1.44 \pm 0.083$ with maximal differences during the methacholine treatment in doses 0 and $4 \mathrm{mg} / \mathrm{kg}-1.9$-fold and 1.4-fold correspondingly (Figure 2A; $p<0.05$ ). The baseline resistance was 2.6 times higher compared to control $(p<0.05)$ in asthma + IL10 shRNA-interference group, but it was significantly lower compared to the asthma group. During methacholine challenge the resistance increased from $0.75 \pm 0.035$ to $1.17 \pm 0.071$ with differences in methacholine doses 8 and $32 \mathrm{mg} / \mathrm{kg}-1.5$-fold higher and on $19 \%$ lower correspondingly compared to OVA-induced asthma group (Figure 2A).
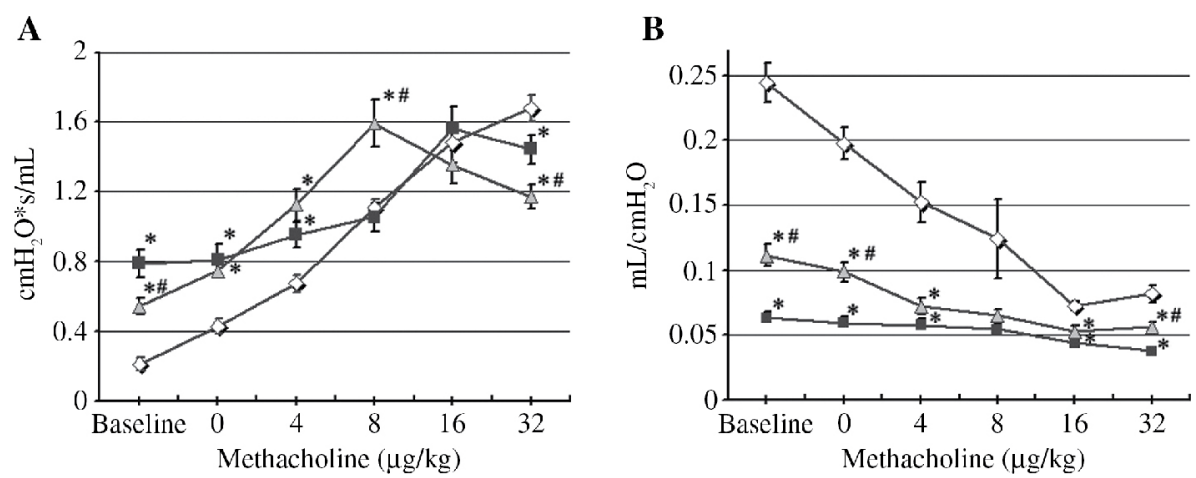

Figure 2: Changes in airway resistance (A) and compliance (B) in control SHR rats ( ), OVA-induced asthma modeling $(\boldsymbol{\square})$ and OVA-induced asthma + IL10 shRNA-interference $(\triangle)$ in methacholine challenge. Values are means \pm SE $(n=7$ per group). * Significant difference compared to control group, $p<0.05$. \# Significant difference between asthma and IL10 shRNA-interference group, $p<0.05$.

Compliance of respiratory system (Crs) in control rats were 4 times $(p<0.05)$ higher compared to OVAinduced asthma group, and was decreased from $0.2 \pm 0.012$ to $0.08 \pm 0.007 \mathrm{~mL} / \mathrm{mmH}_{2} \mathrm{O}$ (Figure 2B). In OVAinduced asthma group Crs was decreased from $0.06 \pm 0.004$ to $0.04 \pm 0.003$ in methacholine test with maximal differences in methacholine doses $0,4,16$ and $32 \mathrm{mg} / \mathrm{kg}(p<0.05)$. RNA-interference significantly influenced on Crs baseline compared to OVA-induced asthma group $(0.11 \pm 0.009$ and $0.06 \pm 0.005$ correspondingly, $p<$ 0.05). During methacholine challenge Crs was decreased in asthma + IL10 shRNA-interference group more prominent than in OVA-induced asthma group (from $0.1 \pm 0.008$ to $0.06 \pm 0.004$ ). Significant differences were determined in methacholine doses 0 and $32 \mathrm{mg} / \mathrm{kg}$ compared to OVA-induced asthma group $(p<0.05)$.

Thus, knockdown of IL10 gene prevents asthma-induced negative changes in baseline parameters of resistance and compliance and ameliorates the decline of compliance during experimental bronchoconstriction (methacholine application). 


\subsection{Percentage of T-Helper 2 Cells in BALF}

In modeling of OVA-induced asthma the quantity of T-helper 2 cell population in BALF was increased in 2.5fold ( $p<0.05)$ compared to control (Figure 3). In rats with RNA interference of IL10 the percentage of T-helper 2 cell presence in BALF was slightly lower compared to OVA-induced asthma group $(p>0.05)$.

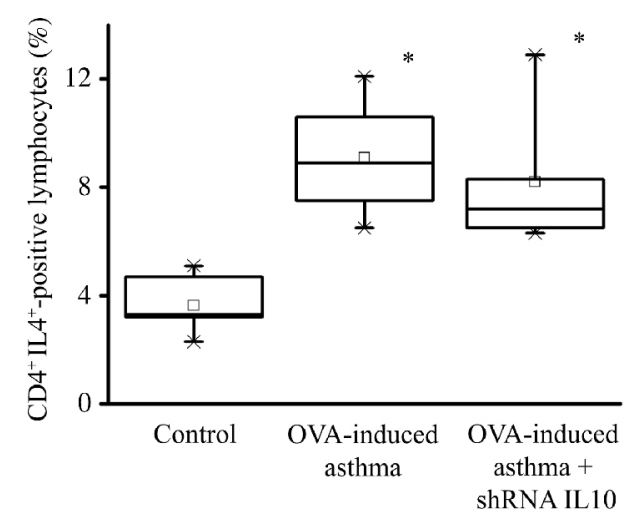

Figure 3: Percentage of T-helper 2 lymphocytes in BALF in control SHR rats, OVA-induced asthma modeling and OVAinduced asthma + IL10 shRNA-interference ( $n=7$ per group). Significant difference compared to control group, $p<0.05$.

\subsection{Morphology}

The lung sections from the control rats were predominantly showed the normal histological architecture of the lung, these samples exhibited only thin interalveolar septa. The wall of bronchioles was contoured by singlelayer of ciliated cuboidal epithelium and a thin layer of smooth muscle fibers (Figure 4). However, slight thickening of the interalveolar septa and negligible peribronchial infiltrates were observed locally in some samples, which can be explained by early manifestations of hypertension in the line of investigated rats (SHR).
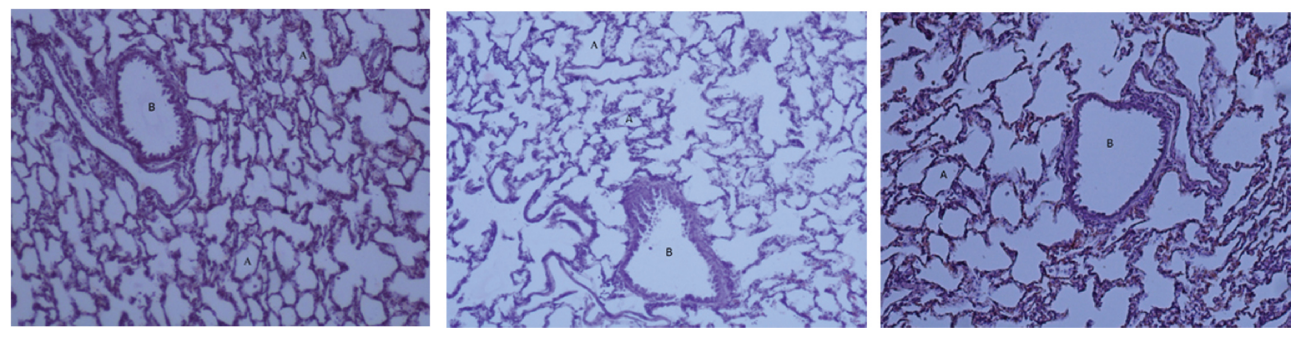

Figure 4: Microscopy of control rat lung slices with a normal lung architecture. Alveoli (A), bronchioles (B), and blood vessels (bv) are indicated, $\times 100$.

In lung sections of rats with OVA-induced asthma the total bronchiole wall was to thicker mostly as a result of peribronchial edema and mononuclear infiltration, bronchial mucosal hyperplasia, as well as increased bronchial smooth muscle mass. Some bronchioles showed intrabronchial cellular debris associated with desquamated epithelium and macrophages. The thickening of interalveolar septa due to red blood cells (RBCs) and mononuclear cellular infiltration, the focal extravasated RBCs in the alveolar spaces up to the collapse of some alveoli with dilatation and ruptures of others, the dilated and congested blood vessels with perivascular edema were observed (Figure 5).
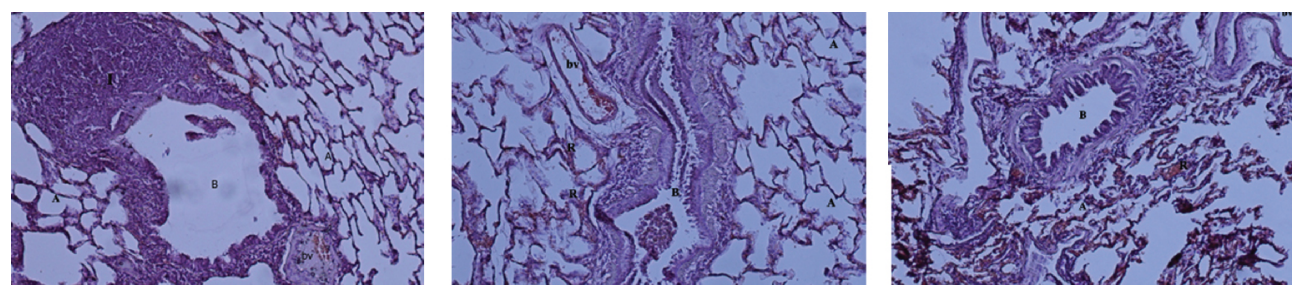

Figure 5: Lung of the OVA-induced asthma modeling rats. Bronchioles with exfoliated epithelial cells in its lumen, mononuclear cellular infiltration surrounding the bronchioles and presented in the interalveolar septa, thickening of interalveolar septa by RBCs are shown. Alveoli (A), bronchioles (B), and blood vessels (bv), mononuclear cellular infiltration (I) are indicated, $\times 100$. 
The IL10 RNA-interference to some extent attenuated inflammatory process in the lung tissue (Figure 6 and Figure 7), and we observed mild alveolar and peribronchial inflammatory cellular infiltration as well as some reduction of thickness of interalveolar septa were shown. The parameters that characterize the airway wall remodeling were slightly reduced both in small and medium bronchioles compared to asthmatic group of animals (Figure 8 and Figure 9).

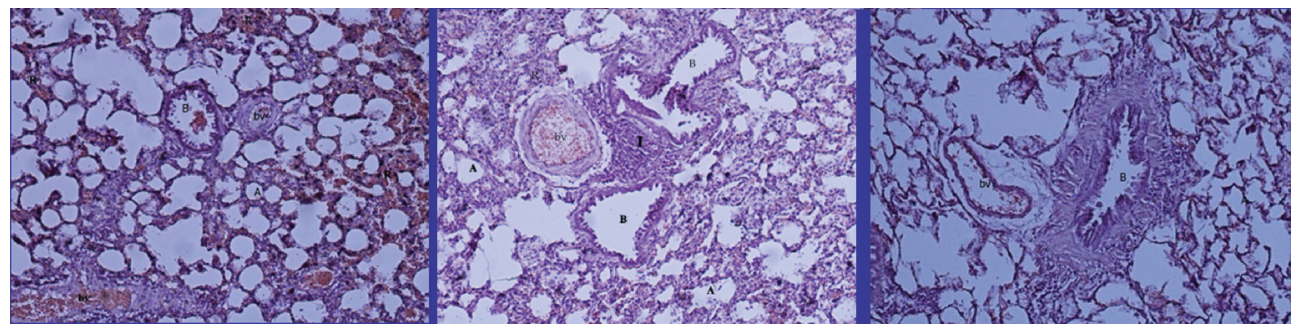

Figure 6: Lung of the OVA-induced asthma + IL10 shRNA-interference rats. Extravasated RBCs and cellular infiltrate in the alveolar spaces with dilatation and rupture of other alveoli is seen. Dilated and congested blood vessel is present. Alveoli (A), bronchioles (B), and blood vessels (bv), mononuclear cellular infiltration (I), extravasated RBCs (R) are indicated, $\times 100$.

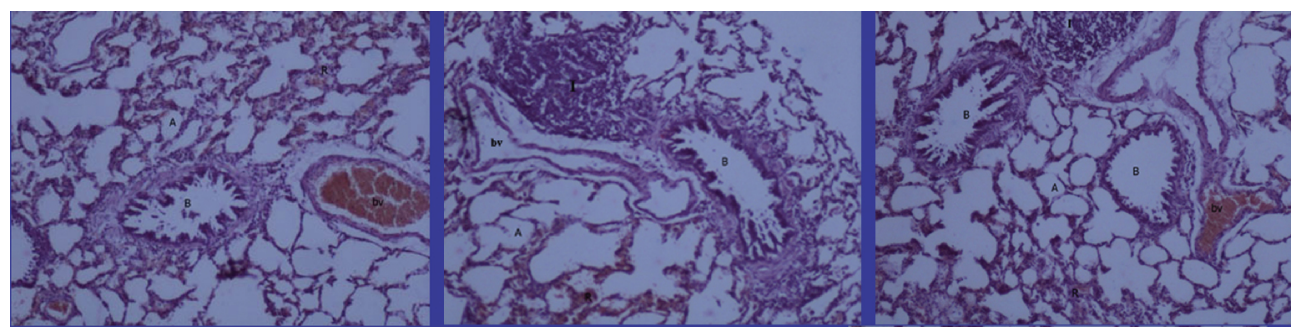

Figure 7: lungs of the OVA-induced asthma + IL10 shRNA-interference rats. Few mononuclear cellular infiltration surrounding the bronchioles and presented in the interalveolar septa, the extravasated RBC and congested blood vessels are seen. Alveoli (A), bronchioles (B), and blood vessels (bv), mononuclear cellular infiltration (I) extravasated RBCs (R) are indicated, $\times 100$.

A

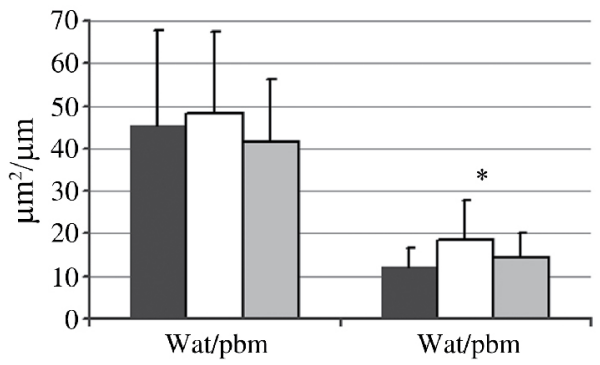

B

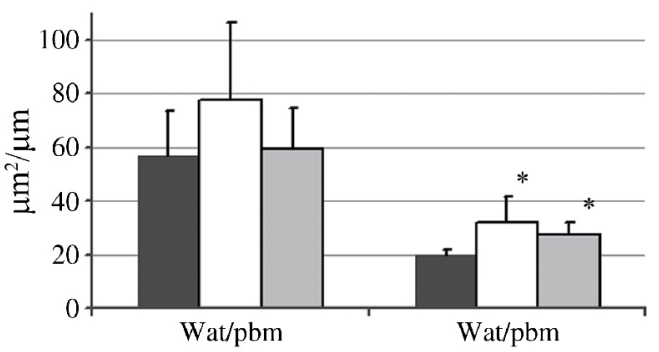

Figure 8: Relation of the total bronchial wall area to the perimeter basement membrane (WAt/Pbm) and the inner wall area to the perimeter basement membrane (WAi/Pbm) in small (A) and medium (B) airways. - control SHR, $\square-$ OVAinduced asthma, $\square-$ OVA-induced asthma + IL10 shRNA-interference. Values are means \pm SD. * significant difference compared to control group, $p<0.05$.
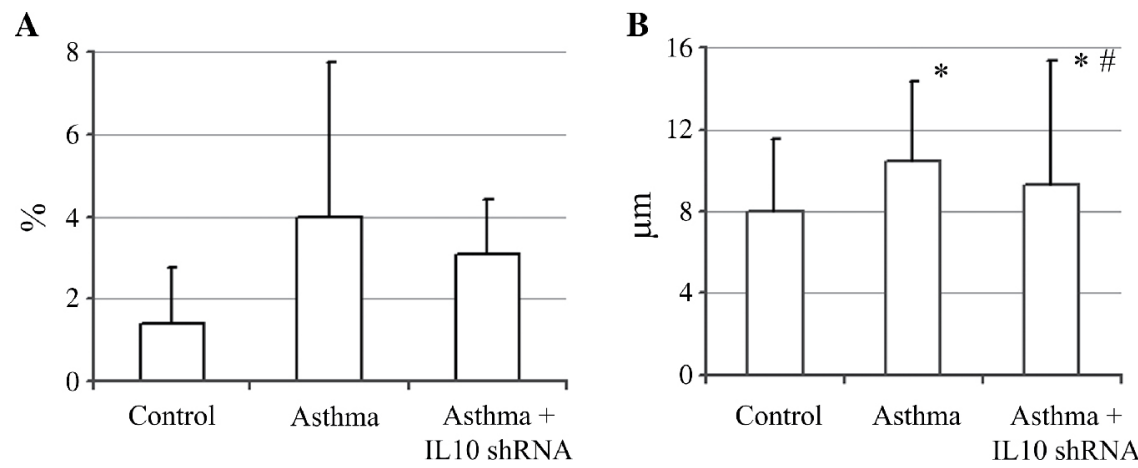

Figure 9: Level of infiltration (A) and thickness of alveoli septum (B) in control SHR, OVA-induced asthma and OVAinduced asthma + IL10 shRNA-interference. Values are means \pm SD. * significant difference compared to control group, $p<0.05$. 


\section{Discussion}

Modeling of comorbid pathology is not routine technique and we have found only few publications in this area. According to our knowledge, we are the first to develop an experimental model of bronchial asthma and arterial hypertension comorbidity presented in the current paper. Earlier Gilmour et al. [21] demonstrated the susceptibility of SHR to the increased inflammation in the lungs while exposed to particle matter compared to Wistar Kyoto rats due to activation of TLR4-mediated signaling. Kodavanti et al. [22] has proved that SHR is a better model for experimental bronchitis, because the sulfur dioxide induces marked immune dysregulation, oxidative stress, impairment of cell signaling, and fatty acid metabolism in SHR compared to Sprague Dawley rats.

Here we provide the data experimentally confirming functionality of the target (IL10) which was in silico predicted to be associated with both asthma and hypertension was confirmed in the experimental model of this comorbid pathology. IL-10 is the well-known cytokine participating in the inflammation of different genesis, can block NF- $\kappa$ B activity and is involved in the regulation of the JAK-STAT signaling pathway. These pathways are involved in both asthma and hypertension pathogenesis. We showed that during RNA-interference of IL10 gene in vivo changes both cardiac and lung function parameters. These changes in the heart work can be described as a positive. But the more intensive heart work (the increase of cardiac output, minute volume, heart work, etc) can lead to exhaust and decompensation of the heart functions comparing to control rats. Knockdown of IL10 gene in asthma modeling induces some positive changes in respiratory functions of asthmatic animals such as decreased elastance and increased compliance of the lungs, as well as less pronounced pathomorphological changes in the lung tissue (for example, the thickness of small and medium bronchioles, characterized bronchial asthma). These data are surprising due to the known function of IL10 as anti-inflammatory cytokine [23], [24] and the decrease in its expression was expected to facilitate asthmatic changes in the lungs. It could be explained by the compensatory increase in the production of other anti-inflammatory substances with stronger effects. Moreover, the present investigation is concerned only the early signs of asthma (the challenge with OVA for only 7 days), and the negative consequences of IL-10 knockdown could appear later. This point definitely needs further investigation. But according some data [25], [26] systemic administration of IL10 for autoimmune therapy proved to be paradoxically proinflammatory. Intravenously plasmid injection in our experiments induces systemic effect also and some positive changes in respiratory parameters in rats with OVA-induced asthma are logically in this context.

Thus, some positive changes in the asthma development in SHR lead to the overload of heart and increases its function, we do not think that decrease of $I L-10$ level or influence on its receptors is the perspective mechanism for the development of new therapeutically approaches for the treatment of asthma and hypertension comorbidity treatment. Still the new model for comorbidity, developed and described in this work can be useful for the further search of other targets.

\section{Acknowledgements}

This study was supported by the Volkswagen Stiftung Trilateral Partnerships - Cooperation Projects between Scholars and Scientists from Ukraine, Russia, and Germany "In silico screening and experimental validation of new drug targets for the treatment of co-morbid multifactorial diseases" (\# 90335).

Conflict of Interest Statement: Authors state no conflict of interest. All authors have read the journal's Publication ethics and publication malpractice statement available at the journal's website and hereby confirm that they comply with all its parts applicable to the present scientific work.

\section{References}

[1] Feinstein AR. The pre-therapeutic classification of co-morbidity in chronic disease. J Chron Dis 1970;23:455-68.

[2] van den Akker M, Buntinx F, Metsemakers ]F, Roos S, Knottnerus ]A. Multimorbidity in general practice: prevalence, incidence, and determinants of co-occurring chronic and recurrent diseases. J Clin Epidemiol 1998;51:367-75.

[3] Kraemer HC. Statistical issues in assessing comorbidity. Stat Med 1995;14:721-33.

[4] Einarson TR, Acs A, Ludwig C, Panton UH. Prevalence of cardiovascular disease in type 2 diabetes: a systematic literature review of scientific evidence from across the world in 2007-2017. Cardiovasc Diabetol 2018;17:83.

[5] Su X, Ren Y, Li M, Zhao X, Kong L, Kang ]. Prevalence of comorbidities in asthma and nonasthma patients: a meta-analysis. Medicine 2016;95:e3459. 
[6] Prosser R, Carleton B, Smith A. The comorbidity burden of the treated asthma patient population in British Columbia. Chronic Dis Inj Can 2010;30:46-55.

[7] Tattersall MC, Guo M, Korcarz CE, Gepner AD, Kaufman JD, Liu K], et al. Asthma predicts cardiovascular disease events. Arterioscler Thromb Vasc Biol 2015;35:1520-5.

[8] Cooke AA, Connaughton RM, Lyons CL, McMorrow AM, Roche HM. Fatty acids and chronic low grade inflammation associated with obesity and the metabolic syndrome. Eur] Pharmacol 2016;785:207-14.

[9] Montecucco F, Pende A, Quercioli A, Mach F. Inflammation in the pathophysiology of essential hypertension. ] Nephrol 2011;24:23-34.

[10] Pietri P, Vlachopoulos C, Tousoulis D. Inflammation and arterial hypertension: from pathophysiological links to risk prediction. Curr Med Chem 2015;22:2754-61.

[11] Chehimi M, Vidal H, Eljaafari A. Pathogenic role of IL-17-producing immune cells in obesity, and related inflammatory diseases. J Clin Med 2017;6:68.

[12] Asghar A, Sheikh N. Role of immune cells in obesity induced low grade inflammation and insulin resistance. Cell Immunol 2017;315:1826.

[13] Xu M, Xu ], Yang X. Asthma and risk of cardiovascular disease or all-cause mortality: a meta-analysis. Ann Saudi Med 2017;37:99-105.

[14] Saik OV, Demenkov PS, Ivanisenko TV, Bragina EY, Freidin MB, Goncharova IA, et al. Novel candidate genes important for asthma and hypertension comorbidity revealed from associative gene networks. BMC Med Genomics 2018;11(Suppl 1):15.

[15] Li M, Shang Y. Inhaled corticosteroids inhibit substance P receptor expression in asthmatic rat airway smooth muscle cells. BMC Pulmonary Medicine 2012;12:79.

[16] Vanacker N], Palmans E, Kips ]C, Pauwels RA. Fluticasone inhibits but does not reverse allergen-induced structural airway changes. Am ] Respir Crit Care Med 2001;163:674-9.

[17] Zhukovska A, Shysh A, Bacova B, Jana R, Tamara B, Csilla V, et al. Heart-protective effect of n-3 PUFA demonstrated in a rat model of diabetic cardiomyopathy. Mol Cell Biochem 2014;389:219-27.

[18] Yousuke F, Shunji H, Takeshi M, Hiroyuki W, Tamaki N, Hideki H, et al. Pulmonary inflammation and cytokine dynamics of bronchoalveolar lavage fluid from a mouse model of bronchial asthma during A(H1N1)pdmo9 influenza infection. Sci Rep 2017;7:1-9.

[19] Chernyshov VP, Sudoma IO, Dons' koi BV, Kostyuchyk AA, Masliy YV. Elevated NK cell cytotoxicity, CD158a expression in NK cells and activated T lymphocytes in peripheral blood of women with IVF failures. Am ] Reprod Immunol 2010;64:58-67.

[20] Palmans E, KIPS ]C, Pauwels RA. Prolonged allergen exposure induces structural airway changes in sensitized rats. Am ] Respir Crit Care Med 2000;161:627-35.

[21] Gilmour PS, Schladweiler MC, Richards ]H, Ledbetter AD, Kodavanti UP. Hypertensive rats are susceptible to TLR4-mediated signaling following exposure to combustion source particulate matter. Inhal Toxicol 2004;16(Suppl 1):5-18.

[22] Kodavanti UP, Schladweiler MC, Ledbetter AD, Ortuno RV, Suffia M, Evansky P, et al. The spontaneously hypertensive rat: an experimental model of sulfur dioxide-induced airways disease. Toxicol Sci 2006;94:193-205.

[23] Ogawa Y, Duru EA, Ameredes BT. Role of IL-10 in the resolution of airway inflammation. Curr Mol Med 2008;8:437-45.

[24] Hawrylowicz CM, O' Garra A. Potential role of interleukin-10-secreting regulatory T cells in allergy and asthma. Nat Rev Immunol 2005;271-83.

[25] Tilg H, VanMontfrans C, Van den Ende A. Treatment of Crohn's disease with recombinant human interleukin 10 induces the proinflammatory cytokine interferon. Gut 2002;2:191-5.

[26] Lauw FN, Pajkrt D, Hack CE, Kurimoto M, Van Deventer S], Van der Poll T. Proinflammatory effects of IL-10 during human endotoxemia. ] Immunol 2000;165:2783-9. 